\title{
Dependence of the quality of adhesion between poly(dimethylsiloxane) and glass surfaces on the composition of the oxidizing plasma
}

\author{
Kenny Chau • Brent Millare $\cdot$ Adam Lin • \\ Srigokul Upadhyayula $\cdot$ Vicente Nuñez $\cdot$ \\ Hong Xu $\cdot$ Valentine I. Vullev
}

Received: 20 August 2010/Accepted: 20 October 2010/Published online: 9 November 2010

(C) The Author(s) 2010. This article is published with open access at Springerlink.com

\begin{abstract}
Controlled surface oxidation of polydimethylsiloxane (PDMS) is essential for permanent adhesion between device components composed of this elastomer. The permanent adhesion between such microdevice components results from covalent crosslinking across the interfaces between PDMS and other silica-based materials, such as glass, quartz, and PDMS. Optimal duration and conditions of oxidation, attained via treatments with oxygen-containing plasma, are crucial for microfabrication procedures with quantitative yields. While insufficient PDMS oxidation does not provide high enough surface density of siloxyl groups for cross-interface linking, overoxidation of PDMS yields rough silica surface layers that prevent the adhesion between flat substrates. Ideally, for a set of plasma conditions, the range of treatment durations producing permanent adhesion should be as broad as possible: i.e., the surface oxidation of PDMS sufficient for irreversible binding has to complete significantly before the effects of overoxidation become apparent. Such a requirement assures that relatively small fluctuations in the treatment conditions will not result in over- or under-oxidation and, hence, will not compromise the yields of the fabrication procedures. We examined the dependence of the quality of adhesion (QA) between
\end{abstract}

K. Chau $\cdot$ B. Millare $\cdot$ A. Lin $\cdot$ S. Upadhyayula $\cdot$ V. Nuñez H. Xu · V. I. Vullev $(\square)$

Department of Bioengineering and the Center for Bioengineering Research, University of California, Riverside,

Bourns Hall A-220, Riverside, CA 92521, USA

e-mail: vullev@ucr.edu

Present Address:

B. Millare

Department of Biomedical Engineering, Johns Hopkins University School of Medicine, 720 Rutland Avenue, Baltimore, MD 21205, USA plasma-treated PDMS and glass substrates on the composition of the oxygen-containing plasma and on the radio frequency (RF) of the plasma generator. We observed that plasma generated at megahertz RF provided superior conditions than kilohertz RF. Concurrently, an increase in the oxygen content of binary gas mixtures, used for the plasma, broadened the treatment durations that afford superior QA.

Keywords Air $\cdot$ Nitrogen $\cdot$ Argon $\cdot$ Capacitively coupled plasma $\cdot \mathrm{CCP} \cdot$ Discharge plasma

\begin{tabular}{ll}
\multicolumn{2}{l}{ Abbreviations } \\
$\varepsilon$ & Engineering strain \\
$\mu \mathrm{FD}$ & Microfluidic device \\
$\sigma$ & Engineering stress \\
PDMS & Poly(dimethylsiloxane) \\
$P_{\mathrm{RF}}$ & Radio-frequency power \\
$\mathrm{QA}$ & Quality of adhesion \\
$\mathrm{RF}$ & Radio frequency \\
$\mathrm{TSM}$ & Tensile-strength measurement
\end{tabular}

\section{Introduction}

This article describes an investigation of the adhesion between polydimethylsiloxane (PDMS) and glass substrates pretreated with oxygen-containing plasma. We examined the dependence of the quality of adhesion on the content of the gas mixture used for the plasma and on the radio frequency (RF) of the plasma generator. Exposure of the substrates to plasma composed of air and of other gas mixtures revealed that the use of pure oxygen for the plasma treatments, at $\mathrm{MHz} \mathrm{RF}$ and $50-\mathrm{W}$ power, provides 
the widest range of optimal conditions for PDMS-glass adhesion.

Low reagent and power consumption, cost-efficient fabrication, and relatively short operating time periods are some of the key advantages of microfluidic devices ( $\mu$ FDs) (Dittrich and Manz 2006; Whitesides 2006; Thomas et al. 2010b). PDMS is a widely used material for fabricating $\mu$ FDs (Gong and Wen 2009; Zhou et al. 2010; Vullev et al. 2006; Thomas et al. 2010a). Despite its shortcomings, such as porosity ( $\mathrm{Li}$ et al. 2009; Mehta et al. 2009; Chueh et al. 2007; Shin et al. 2003) and susceptibility to a broad range of organic solvents (Lee et al. 2003), PDMS is biocompatible (Belanger and Marois 2001; Zhuang et al. 2007) and allows for expedient and facile reproduction of features with nanometer precision that are essential for $\mu$ FDs (Gates 2005; Cong and Pan 2008).

The fabrication of microdevices, comprising PDMS components, involves controlled oxidation of the dimethylsiloxane surfaces to ensure a permanent adhesion to other silica-based substrates (Millare et al. 2008; Bhattacharya et al. 2005; Hui et al. 2005). Oxidative conversion of the methylsiloxane to siloxy functionality $\left(\mathrm{Si}-\mathrm{CH}_{3} \rightarrow \mathrm{Si}-\mathrm{O}^{-}\right.$) provides means for covalent crosslinking between the two substrates, when brought in contact (Hui et al. 2005; Morra et al. 1990; Chaudhury and Whitesides 1992). A nucleophilic siloxy oxygen from one of the substrates attacks an electrophilic silicon from the other, forming a covalent bond across the interface between the two materials. For example, a noncovalent reversible adhesion between PDMS and glass, essential for a range of patterning fabrication steps (Hong et al. 2008), does not sustain stress exceeding about $10 \mathrm{kPa}$ (Millare et al. 2008). The interfacial covalent enhancement of the PDMS-glass adhesion, however, can sustain stress of $1 \mathrm{MPa}$ or larger (Millare et al. 2008).

Recently, we examined the changes that treatments with plasma, composed solely of oxygen, induced on the PDMS surface (Millare et al. 2008). The surface properties of the plasma-treated siloxane elastomer correlated well with its ability to adhere permanently to glass substrates (Millare et al. 2008). On the other hand, the use of oxidizing plasma composed of air or of other oxygen-containing gas mixtures, instead of pure oxygen, is a prevalent approach in the PDMS surface activation for the fabrication of $\mu$ FDs (Gou et al. 2010; Anderson et al. 2000; McDonald and Whitesides 2002). Furthermore, while our initial studies were conducted for plasma generated at $\mathrm{MHz} \mathrm{RF}$, cost restrictions often drive the choices for equipping soft-lithography labs with $\mathrm{kHz}$ plasma generators.

For the $\mathrm{kHz}$ range, commercially available choices of frequencies for RF plasma generators appear relatively broad. Plasma generators, operating in the $\mathrm{GHz}$ frequency domain, became widely available as a "spin off" of the microwave-oven technology. For the $\mathrm{MHz}$ range, however, the prevalent development of plasma generators operating at $13.56 \mathrm{MHz}$ and its harmonics was driven by a regulation from the Federal Communication Commission.

Herein, we studied the dependence of the quality of PDMS-glass adhesion on the composition of the oxidizing plasma. For air plasma, an increase in the RF from $40 \mathrm{kHz}$ to $13.65 \mathrm{MHz}$ (1) improved the reproducibility of the optimal adhesion and (2) narrowed the acceptable range of conditions for PDMS-glass adhesion and shifted it to shorter treatment time durations. Tests with nitrogenoxygen and argon-oxygen plasma mixtures, however, revealed that the use of $100 \%$ oxygen for the plasma treatments at intermediate $\mathrm{RF}$ power $(\sim 50 \mathrm{~W}, 13.56 \mathrm{MHz})$ provides the widest range of optimal conditions for PDMSglass adhesion.

\section{Results and discussion}

\subsection{Plasma treatments and quality of adhesion}

For the oxidative treatment of the PDMS and glass substrates, we employed capacitively coupled discharge plasma systems, equipped with RF generators operating at $40 \mathrm{kHz}$ and $13.56 \mathrm{MHz}$. The color of the light, emitted by the ionized gas in the plasma chamber, is an important observable for monitoring the quality of the plasma. (Caution: The intense radiation from the gas plasma may cause permanent eye damage. While operating it, avoid looking directly into the plasma chamber without appropriate protective eyewear.) Plasma of pure oxygen appeared white, while plasma of dry air was pink (Fig. 1). Furthermore, for plasma generated at $40 \mathrm{kHz}$, the colors were less intense than the colors of the plasma generated at 13.56 MHz.

The oxidative treatment of the PDMS slabs and of the glass slides involved exposing them to the low-pressure gas plasma for different time durations. Immediately after the treatment, we brought into contact the plasma-exposed PDMS and glass surfaces (contact area $\sim 1 \mathrm{~cm}^{2}$ ) and cured them overnight at $120^{\circ} \mathrm{C}$ under vacuum $(340 \mathrm{mbar}$ ). By applying pulling force, in the direction of lifting the PDMS from the glass slides, we examined the quality of adhesion (QA). The values of QA were estimated from ternary semiquantitative evaluation of PDMS-glass adhesion (Millare et al. 2008): i.e., $\mathrm{QA}=2$ for permanent adhesion throughout the whole contact area; $\mathrm{QA}=1$ for partial adhesion (i.e., the regions of permanent adhesion are randomly distributed over the contact area); and QA $=0$ for lack of any permanent adhesion. The QA for each set of the plasma composition and for plasma-treatment conditions was calculated by averaging the values of QA for all samples pretreated under these conditions. 

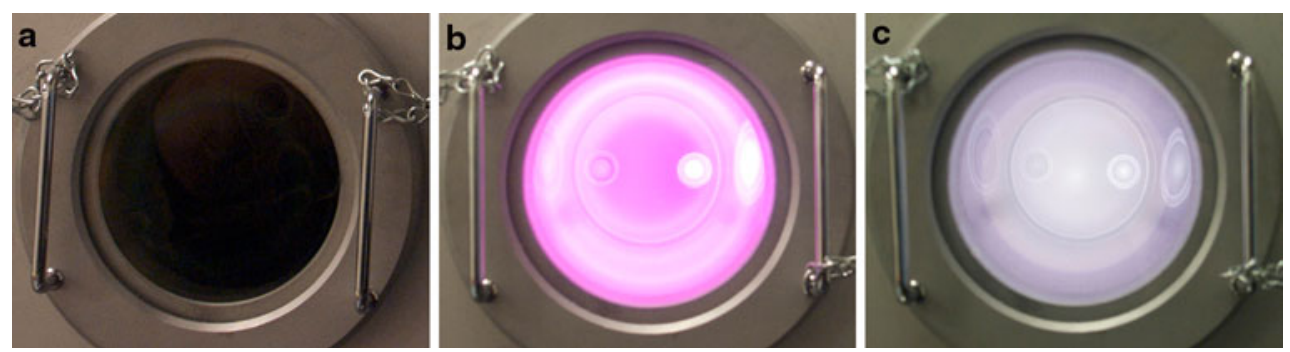

Fig. 1 External view of the plasma chamber: a prior to ignition of the plasma; $\mathbf{b}$ with air plasma ignited; and $\mathbf{c}$ with pure-oxygen plasma ignited (plasma conditions: $\mathrm{RF}=13.56 \mathrm{MHz}$; power $=50 \mathrm{~W}$; and pressure $=0.35 \mathrm{mbar}$ )

To characterize the strength of the adhesive bonds between PDMS and glass, we employed tensile-strength measurements (TSMs) on PDMS slabs adhered to two glass slides (Fig. 2a). The materials at one of the PDMSglass interface were permanently adhered, i.e., $\mathrm{QA}=2$, while at the other PDMS-glass interface, QA was 0,1 , or 2.

When the quality of adhesion at both PDMS-glass interfaces was 2, the samples sustained tensile stress of about $1 \mathrm{MPa}$ or more (corresponding to $100 \mathrm{~N}$ for $1 \mathrm{~cm}^{2}$ interfaces). Instead of breaking at the PDMS-glass interfaces, in all tests, the failure of the junction involved tearing the PDMS slabs, breaking the glass, or separating the glass-wood-glued interfaces (Millare et al. 2008). Apparently, due to the mechanical properties of the materials, these results did not allow to quantify the ultimate tensile strength of permanently adhered PDMS-glass interfaces. PDMS-glass bound with QA $=2$ was stronger than the intra-polydimethylsiloxane bonds, i.e., PDMS fractured before the adhesive bond between PDMS-glass fails. Nevertheless, our findings are indicative that $\mathrm{QA}=2$ corresponds to interfacial strength that exceeds $1 \mathrm{MPa}$. The measured stress, at which PDMS-glass junctions separate (i.e., above $1 \mathrm{MPa}$, which corresponds to $145 \mathrm{psi}$ ), tends to exceed the values of the bond strength reported for PDMS adhesion to other substrates (Bhattacharya et al. 2005). This finding suggests that such previously reported values reflect incomplete adhesion throughout the surface area (i.e., $\mathrm{QA}=1$ ) or the use of polymer with lesser strength.

In contrast, for $\mathrm{QA}=0$, the PDMS-glass interface readily dissociated under relatively small stress: i.e., at $\sigma<10 \mathrm{kPa}$, corresponding to $1 \mathrm{~N}$ tensile force for $1 \mathrm{~cm}^{2}$ (Fig. 2b, inset). Unlike the dissociation of interfaces adhered with $\mathrm{QA}=2$ (or $\mathrm{QA}=1$ ), the dissociation of
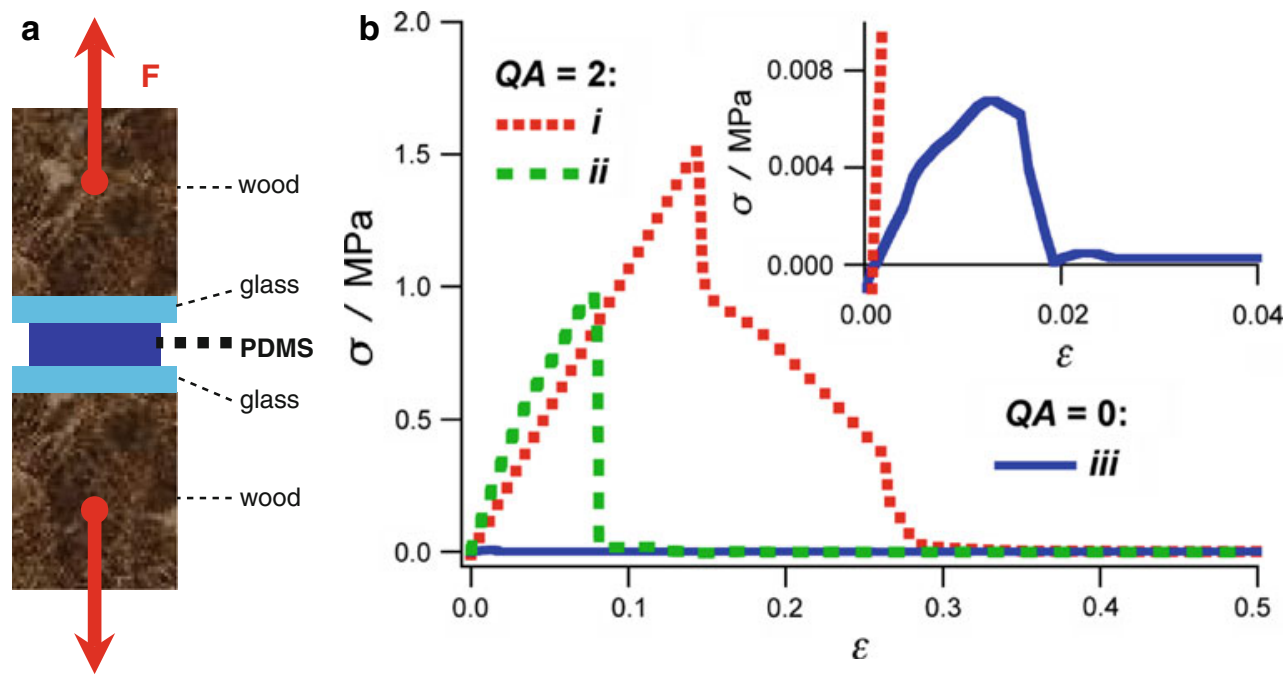

Fig. 2 Tensile-strength measurements (TSMs) of PDMS-glass adhesion. a Scheme of the wood/glass/PDMS/glass/wood junction used for the TSMs. b Tensile stress/strain, $\sigma / \varepsilon$, relation for QA $=2$ (curves $\mathrm{i}$ and ii), and $\mathrm{QA}=0$ (curves iii). For curve i, both the permanent adhesion at both PDMS-glass interfaces was achieve by 30 -s treatments with air plasma, $P_{\mathrm{RF}}=50 \mathrm{~W}$ and $\mathrm{RF}=13.56 \mathrm{MHz}$. For curve ii, both interfaces were treated with oxygen plasma under identical power, RF and time settings. For curve iii, one of the
PDMS-glass interfaces was treated under identical conditions as the samples for curve $i$ and allowed to age for a day. Then at the other PDMS-glass interface, the materials were brought in contact without any plasma treatment to ensure $\mathrm{QA}=0$. The failure point of $i$, at about 1.5 MPa, was a result of tearing the PDMS slab, while the failure point of ii resulted from breaking one of the glass slides. No material deterioration was observed for iii after the disjointment at $\sim 7 \mathrm{kPa}$ 
PDMS-glass interface held together at $\mathrm{QA}=0$ did not leave visible debris of PDMS material on the glass surfaces. This observation, along with the differences in the interfacial strengths exceeding two orders of magnitude, provided the discernibility and the semi-quantitative definitions for $\mathrm{QA}=0$ and $\mathrm{QA}=2$.

Another important distinction between PDMS-glass interfaces with $\mathrm{QA}=0$ and $\mathrm{QA}=2$ was in their susceptibility to peeling forces. (In contrast to the tensile forces, applied orthogonally and equally distributed over the interface area, peeling forces are applied only at the edge of the PDMS-glass interface and involve bending of the elastomer slab to lift it off the glass surface.) For QA $=0$, the PDMS slabs readily peeled off the glass slides under forces of about a Newton or a fraction of a Newton. (The interfaces with $\mathrm{QA}=0$ would occasionally dissociate even by the weight of the glass slide upon lifting the PDMS slab.) For QA $=2$, attempts to peel the PDMS from the glass led to tearing the polymer (under excessive forces) rather than separating it from the glass slides.

As an intermediate, $\mathrm{QA}=1$ was assigned for interfaces, for which the PDMS slabs could be partially lifted off the glass surface and at the same time had regions of permanent adhesion. Attempts to completely peel off such partially bound interfaces resulted in tearing the PDMS slabs and leaving polymer debris onto the glass surfaces. TSMs for interfaces with $\mathrm{QA}=1$, indeed, produced intermediate forces of junction failure, i.e., between about 20 and $60 \mathrm{~N}$. Because of the heterogeneous distribution of the permanently bonded regions throughout such PDMS-glass interfaces, however, we abstained from ascribing tensilestress value for samples with $\mathrm{QA}=1$ (i.e., we measure the forces, but we cannot unambiguously define the surface area of the permanent bonding between the substrates in order to calculate the engineering stress). Similar to $\mathrm{QA}=2$, the separation of the junctions with $\mathrm{QA}=1$ during TSMs involved tearing the PDMS material and leaving polymer debris on the glass surfaces.

As we have demonstrated, the values of QA correlated well with the properties of the oxidized PDMS surfaces (Millare et al. 2008). The underlying reasons for using QA for evaluation of the conditions of PDMS oxidation included (1) the observable property of QA was how well the substrates adhere, rather than surface characteristics that correlate with the adhesiveness of the materials; (2) by avoiding relatively large pulling forces, QA accounts for the bonding between the substrates, rather than for mechanical failures (i.e., tearing) of the polymer; and (3) the measurements required for QA are significantly more expedient than atomic-force microscopy and wetting contact angle goniometry, making such QA estimation the preferred approach for expedient evaluation of a large number of conditions.
The duration of treatment with oxygen-containing plasma is a crucial parameter in establishing optimal conditions that afford high QA in a reproducible manner (Millare et al. 2008). An increase in the extent of PDMS oxidation increases the amount of surface siloxyls and, hence, increases the covalent crosslinking across the substrate interface producing the permanent adhesion. Extensive oxidation of PDMS, however, results in the formation of rough surface layers of silica that prevent adhesion of the over-treated elastomer to other substrates (Millare et al. 2008; Bar et al. 2001).

Initial plasma oxidation produces surface layers (with thickness of about 100-200 nm), in which the silicon atoms are connected with about three or four oxygens. Upon further plasma treatment, the oxidized surface layers become thinner due to the formation of silicon oxide, which has smaller specific volume than the polymer structures (Hillborg et al. 2000). The partially oxidized PDMS surface layers, in which the polymer structure is at least partially preserved, are essential for permanent adhesion. In addition to sufficient number of sites for covalent cross-linking across the interfaces between silica-type materials, such partially oxidized PDMS surface layers still have the smoothness and elasticity needed for establishing practically a complete contact when pressed against the surfaces of the other substrates.

The hydrophilic nature of such partially oxidized PDMS surfaces makes them unstable when exposed to hydrophobic medium, such as air (Morra et al. 1990; Murakami et al. 1998). Within hours, conformational rearrangements pull the partially oxidized polymer chains into the PDMS bulk and/or low-molecular weight nonoxidized polymer chains diffuse to the surface (Eddington et al. 2006) leading to (1) surfaces covered with non-oxidized PDMS chains, (2) recovery of the surface hydrophobicity, and (3) loss of the PDMS adhesiveness. Therefore, for all our studies, we bring the oxidized PDMS and glass substrates in contact with each other within $10-30 \mathrm{~s}$ after the plasma treatments. Because the kinetics of aging of the oxidized PDMS is quite slow, the use of freshly plasma-treated substrates assures that the observed losses of adhesiveness, i.e., low QA values, are not due to conformational rearrangement of the polymer chains at the interfaces between the substrates. Low quality of adhesion, i.e., QA $=0$ and 1 , therefore, is ascribed either (1) to insufficient oxidation and insufficient surface density of siloxy groups essential for interfacial covalent cross-linking between the substrates or (2) to overoxidation and formation of rough brittle layers of silica that prevent the establishment of atomiclevel complete contact throughout the interface between the substrates. 


\subsection{Air plasma generated at different frequencies}

Treatment with air plasma is one of the most frequently used approaches for oxidative activation of PDMS surfaces, making them adhesive to silica-based substrates. We examined the dependence of QA on the duration of treatment of PDMS with air plasma generated at $40 \mathrm{kHz}$ and $13.56 \mathrm{MHz}$ at $\mathrm{RF}$ power, $P_{\mathrm{RF}}$, ranging from 10 to $90 \mathrm{~W}$ (Fig. 3).

For $\mathrm{kHz}$ and $\mathrm{MHz}$ frequencies, an increase in the $\mathrm{RF}$ power shifted the range limits of treatment durations, producing acceptable QA, to shorter times (Figs. 4, 5). For $40 \mathrm{kHz}$, for example, the region corresponding to $\mathrm{QA} \geq 1.7$ shifted from $10-110 \mathrm{~s}$, for $P_{\mathrm{RF}}=30 \mathrm{~W}$, to 10-40 s, for $P_{\mathrm{RF}}=90 \mathrm{~W}$ (Figs. 4a, 5a). Similarly, for $13.56 \mathrm{MHz}$, the region corresponding to $\mathrm{QA} \geq 1.9$ shifted from $20-120 \mathrm{~s}$, for $P_{\mathrm{RF}}<20 \mathrm{~W}$, to $4-80 \mathrm{~s}$, for $P_{\mathrm{RF}}>$ $80 \mathrm{~W}$ (Figs. 4b, 5b). This observation is consistent with the increase in the energy of the ionized gas molecules caused
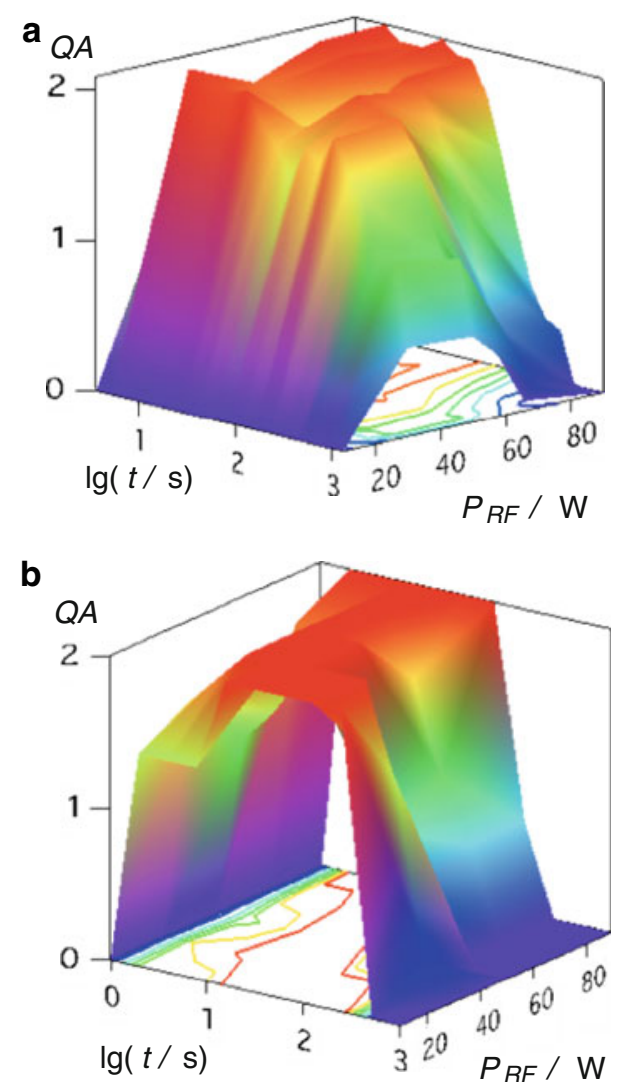

Fig. 3 Surface plots of the quality of adhesion (QA) between poly (dimethylsiloxane) and glass pretreated for various time durations (time, $t$, in seconds is presented in a logarithmic scale), with air plasma generated using different $\mathrm{RF}$ power at: a $40 \mathrm{kHz}$ and b $13.56 \mathrm{MHz}$ (under plasma pressure $=0.35 \mathrm{mbar}$ ). $\mathrm{QA}=2$ corresponds to permanent adhesion, $\mathrm{QA}=1$ corresponds to permanent adhesion, partially distributed throughout the contact interfaces, and $\mathrm{QA}=0$ corresponds to reversible or no adhesion by an increase in the RF power. An increase in the energy of the species colliding with the PDMS surfaces increases the rate of oxidation.

Comparison between the plasma treatments conducted at different frequencies revealed that the use of $13.56 \mathrm{MHz}$ $\mathrm{RF}$ generator provided means for attaining $\mathrm{QA}=2$ in a quantitative and reproducible manner (Figs. 4, 5). At $\mathrm{RF}=13.56 \mathrm{MHz}$, the optimal treatment-duration range was, indeed, narrower than the treatment-duration range resulting in acceptable $\mathrm{QA}$ for $\mathrm{RF}=40 \mathrm{kHz}$ (taking $\mathrm{QA}=1.7$ for a criterion, for example). The treatment at 13.56 MHz, however, provided a well-defined range of conditions (yielding, for example, $\mathrm{QA} \geq 1.9$ ) allowing for permanent adhesion with quantitative yields (Fig. 5b) essential for fabrication procedures.

An important distinction between the $\mathrm{kHz}$ and $\mathrm{MHz}$ treatment appeared to be the rate of oxidation. In comparison with the $\mathrm{MHz}$ conditions, the oxidation appeared overall slower for the $\mathrm{kHz}$ treatments: e.g., for $\mathrm{RF}=$ $40 \mathrm{kHz}$, longer treatment durations were necessary to observe permanent adhesion (Fig. 5a). Concurrently, for the $\mathrm{kHz}$ treatments, QA deteriorated relatively gradually, even manifesting trends of QA decline prior to attaining permanent adhesion with quantitative yields (Figs. 4a, 5a). These findings suggest that for $\mathrm{RF}=40 \mathrm{kHz}$, the trends of overoxidation became apparent prior to the oxidative formation of siloxyls with sufficient surface density. In contrast, treatments at $13.56 \mathrm{MHz}$ revealed well-defined regions of conditions (duration and RF power) that produced QA $=2$ with quantitative yields (Fig. 5b).

We ascribe this frequency-induced discrepancy in quantitatively attaining permanent adhesion to the difference in the energy distribution of the ionized species generated with different RF field. Most of the potential drop in the plasma chamber (and hence most of the transfer of the RF power to the gas) occurs across the millimeter thick gas sheath at the electrode surfaces (Hopwood and Mantei 2003; Jacob 1998; Kawamura et al. 1999). The residence time of the gas molecules within the sheath region is in the order of $10^{-6} \mathrm{~s}$. Therefore, molecules at the sheath exposed to $\mathrm{RF} \ll 1 \mathrm{MHz}$ experience excitation similar to a DC jolt and leave the electrode surface at a different phase of the oscillating field. Such excitation, therefore, produces ionized species with a broad distributions of energies (Kawamura et al. 1999).

In contrast, gas molecules at the electrode surface exposed to $\mathrm{RF} \gg 1 \mathrm{MHz}$ experience excitation that proceeds over several cycles of field oscillation, resulting in ionized species with relatively narrow energy distribution (Kawamura et al. 1999). For $\mathrm{RF}=13.56 \mathrm{MHz}$, therefore, the narrow energy spectrum of the plasma, allowed for reliable attainment of siloxyls with sufficient surface density on the PDMS substrate, prior to overoxidation 
Fig. 4 Dependence of quality of adhesion (QA) between PDMS and glass on the time duration (logarithmic representation) of treatment with air plasma generated using different RF power at: a $40 \mathrm{kHz}$ and $\mathbf{b} 13.56 \mathrm{MHz}$ (under plasma pressure $=0.35$ mbar). The markers represent the experimentally determined data points. The lines represent double-sigmoid data fits. Large error bars reflect fluctuations in the QA outcome for the particular plasma treatment conditions and duration. The error bars are not displayed if they are smaller than the markers
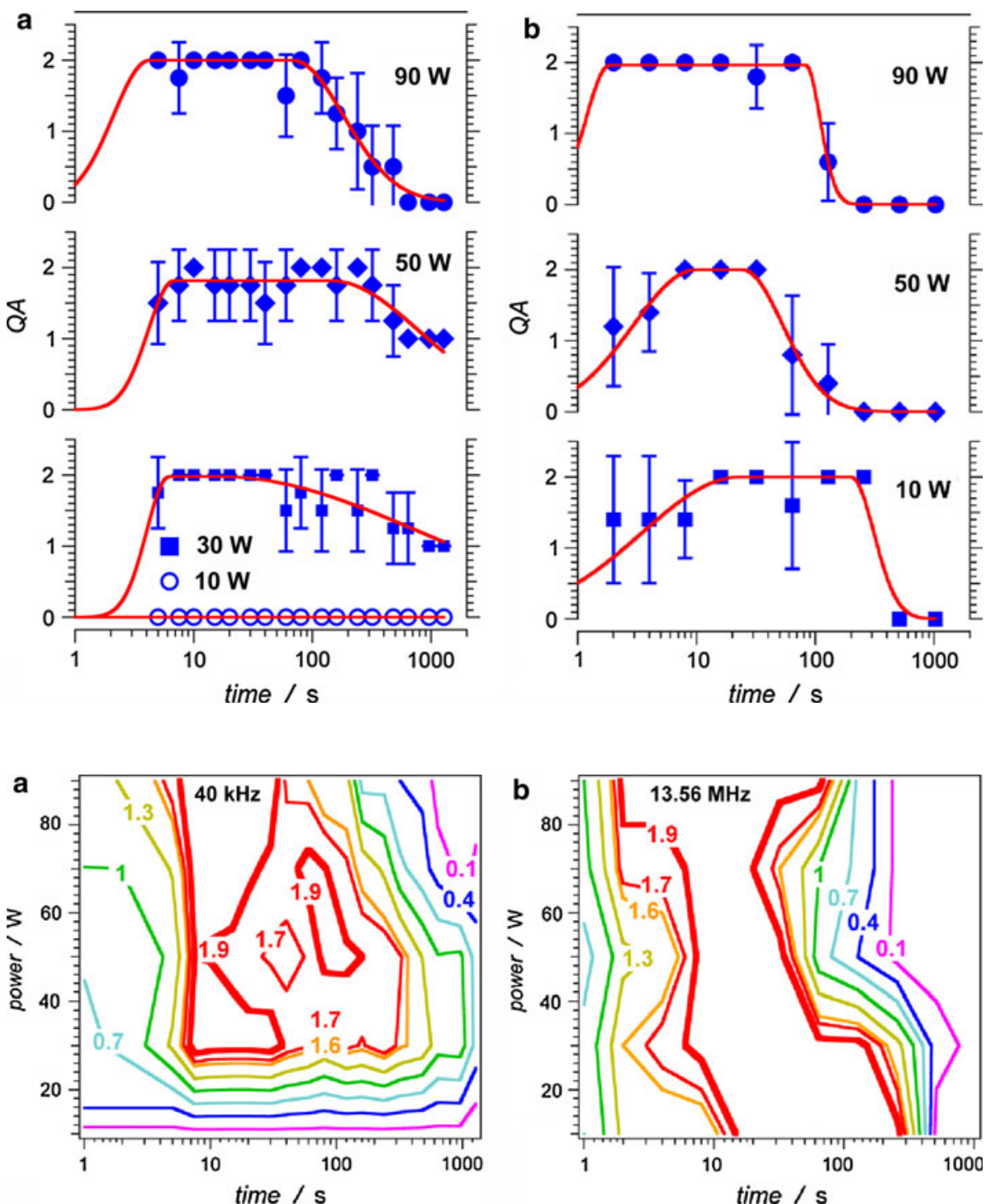

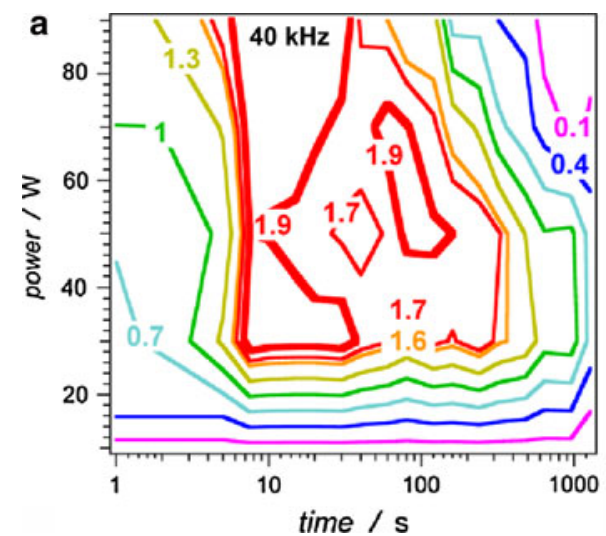

Fig. 5 Contour plots representing the dependence of quality of adhesion (QA) on the $\mathrm{RF}$ power and on the time duration of treatment (logarithmic representation) with air plasma generated under 0.35 mbar pressure at: a $\mathrm{RF}=40 \mathrm{kHz}$ and b $\mathrm{RF}=13.56 \mathrm{MHz}$. The contours corresponding to $\mathrm{QA}=1.7$ and 1.9 surround regions of conditions that can prove feasible for fabrication procedures

\section{.} . 
means for establishing conditions for plasma treatment that lead to outcomes with improved reproducibility.

We examined the dependence of QA on the content of oxygen-nitrogen and oxygen-argon mixtures for plasma treatments of PDMS. In our previous study, we determined that treatment with oxygen plasma generated at $50 \mathrm{~W}$ 13.56 MHz RF proved optimal for obtaining permanent PDMS-glass adhesion (Millare et al. 2008). Therefore, for oxygen-nitrogen and oxygen-argon mixtures we used $P_{\mathrm{RF}}=50 \mathrm{~W}$ and $\mathrm{RF}=13.56 \mathrm{MHz}$.

Because air is composed of $20.9 \% \mathrm{O}_{2}$ and $78.1 \% \mathrm{~N}_{2}$, we hypothesized that plasma treatments with mixtures that contain $20 \%$ or more oxygen would result in QA values similar to the QA we observed for the air treatments. Our findings (for $\mathrm{RF}=13.56 \mathrm{MHz}$ and $P_{\mathrm{RF}}=50 \mathrm{~W}$ ), however, suggested for rejection of this hypothesis (Figs. 6, 7). For plasma treatments with argon-oxygen and for nitrogen-oxygen mixtures, we did not observe quantitative permanent adhesion (i.e., $\mathrm{QA} \geq 1.9$ ) at oxygen content smaller than about 50\% (Fig. 7).

Currently, we do not have a good understanding about the origin of this discrepancy; however, our findings

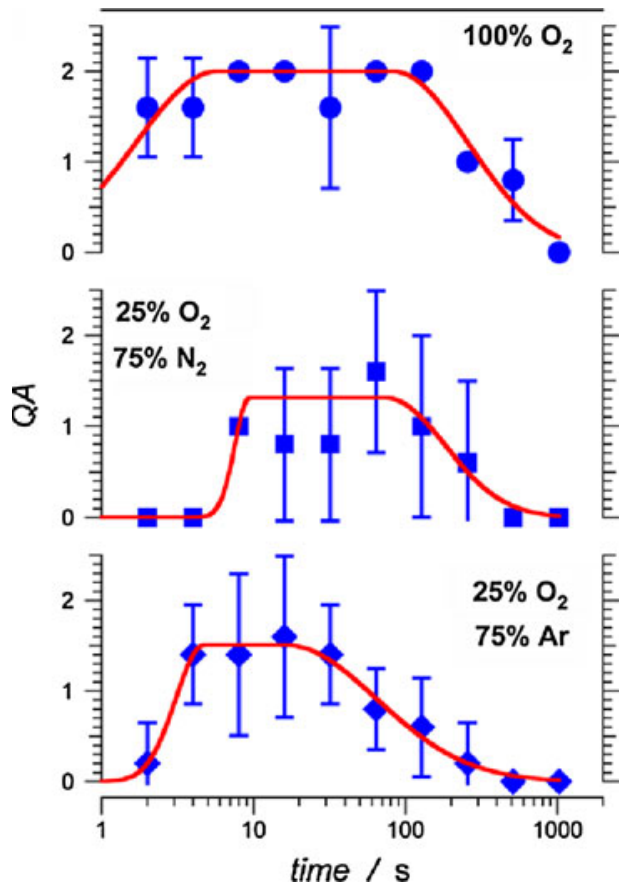

Fig. 6 Dependence of quality of adhesion (QA) between PDMS and glass on the time duration (logarithmic representation) of treatment with different oxygen-containing plasma mixtures generated using $50 \mathrm{~W}$ RF power at $13.56 \mathrm{MHz}$ (under plasma pressure $=0.35 \mathrm{mbar}$ ). The markers represent the experimentally determined data points. The lines represent double-sigmoid data fits. Large error bars reflect fluctuations in the QA outcome for the particular plasma treatment conditions and duration. The error bars are not displayed if they are smaller than the markers suggest that some of the minor components of atmospheric air could be crucial for the oxidative plasma treatments. In fact, some of our initial studies revealed that impurities of air (resultant from employing only one or two degassing cycles) appeared to improve the QA for treatment with plasma mixtures with low oxygen content. For example, traces of air trapped in the PDMS slabs and desorbed into $100 \%$ argon plasma mixture resulted in partial adhesion at treatments exceeding $2 \mathrm{~min}$. Due to the porous nature of PDMS, which is capable of trapping gas, we established a protocol in which we degassed the plasma chamber for prolonged time with increased number of cycles (i.e., pumping the gas down to pressure under $\sim 20 \mu \mathrm{Bar}$ and filling the chamber with the gas mixture of choice).

The trends of dependence of QA on the composition of the plasma-gas mixtures demonstrated that for reproducible adhesion with quantitative yields, treatments with $100 \%$ oxygen provide the most desirable outcomes (Fig. 7). As an oxidative mixture, air is more accessible than pure oxygen. For $P_{\mathrm{RF}}=50 \mathrm{~W}$, however, the range of time durations of oxygen-plasma treatments that yield optimal QA, was wider than the feasible time durations for treatment with air plasma (Fig. 4b middle curve vs. Fig. 6 top curve). Along with this consideration, the uncertainty due to the atmospheric conditions makes the use of pure oxygen a preferred choice for PDMS oxidative plasma treatments.

\subsection{Conditions of oxidative plasma for achieving permanent adhesion}

Three characteristics of QA govern the choice for optimal treatment conditions in order to quantitatively achieve permanent adhesion (e.g., QA > 1.9): (1) the value of QA, averaged from measurements, should be 2 or close to 2 , in order to ensure high quality of adhesion; (2) the error limits for each set of measurements should be zero or close to zero, in order to ensure reproducibility; and (3) the neighboring data points (along the time, $P_{\mathrm{RF}}$, or gas-composition axes) should have QA close to 2 and error limits close to zero, in order to ensure that the outcome would not susceptible to fluctuations in the treatment conditions.

For air plasma generated at $\mathrm{kHz} \mathrm{RF}$, for example, the analysis showed a random distribution of regions for which QA $>1.9$ (Fig. 5a). Closer examination of the individual measurements indicated wide ranges of conditions yielding relatively large average QA values (e.g., QA > 1.7), which, conversely had considerable error limits due to mixed outcomes of $\mathrm{QA}=1$ and 2 (Fig. 4a). According to our observation, for air plasma formed in $40-\mathrm{kHz}$ generators (which is one of the most popular lab setups for PDMS oxidative treatment), the RF power should be at least 20 or $30 \mathrm{~W}$ (and probably not much above $100 \mathrm{~W}$ ) and the 


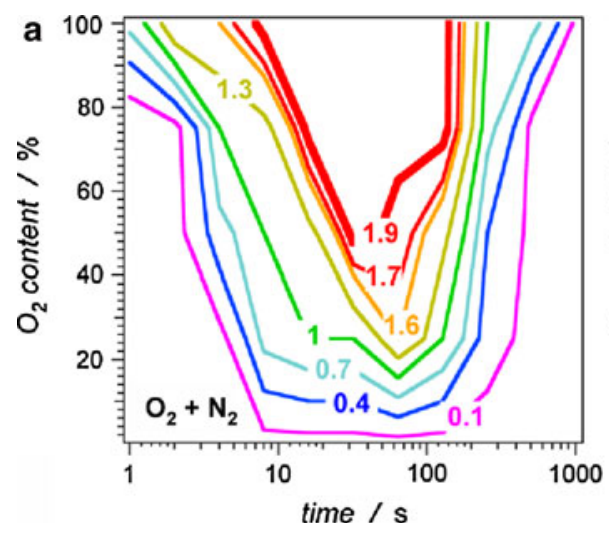

Fig. 7 Contour plots representing the dependence of quality of adhesion (QA) on the oxygen content and on the time duration (logarithmic representation) of treatment with plasma composed of: a oxygen and nitrogen and $\mathbf{b}$ oxygen and argon (plasma

exposure of the polymer to the ionized gas should not be longer than about $40 \mathrm{~s}$, in order to ensure permanent adhesion (Table 1).

While for air plasma generated at $\mathrm{MHz} \mathrm{RF}$, the regions of outcomes with relatively large QA values were narrower that those for $40 \mathrm{kHz}$ (Fig. 5), the continuous set of power/ time conditions that produce QA $>1.9$ was quite well defined (Figs. 4b, 5b). Our findings indicated that air plasma generated at $10 \mathrm{~W} 13.56 \mathrm{MHz}$ RF, yielded permanent PDMS-glass adhesion when the substrates were treated for periods between 20 and $200 \mathrm{~s}$ (Fig. 5b). An increase in the RF power to about $100 \mathrm{~W}$, shortened the upper limit of the treatment duration that reproducibly yielded permanent adhesion to about a minute (Table 1).

Although our findings for air-plasma treatments exhibited reliable reproducibility at $P_{\mathrm{RF}}=90 \mathrm{~W}, 13.56 \mathrm{MHz}$ RF (Fig. 4b, upper curve), we are cautious to make recommendations for treatment durations shorter than about $10 \mathrm{~s}$. Shimming the shunt, $C_{\mathrm{Sh}}$, and the series capacitors, $C_{\mathrm{Sr}}$, which are components of the impedance-matching modules of the plasma systems, in order to maximize the power transferred to the gas plasma (Salem et al. 1998; Singh and Roy 2007) may require up to about 5 s. Even

Table 1 Optimal plasma-treatment conditions for QA $>1.9$

\begin{tabular}{lcll}
\hline Gas & $\mathrm{RF} / \mathrm{MHz}$ & $P_{\mathrm{RF}} / \mathrm{W}$ & Time/s \\
\hline Air $^{\mathrm{a}}$ & 0.04 & $>30$ & $10-100$ \\
Air $^{\mathrm{a}}$ & 13.56 & $10-30$ & $20-200$ \\
& & $40-60$ & $10-50$ \\
& & $80-90$ & $10-60$ \\
Oxygen & 13.56 & 50 & $10-100$
\end{tabular}

Pressure $=0.35$ mbar

${ }^{\text {a }}$ Plasma color-pink

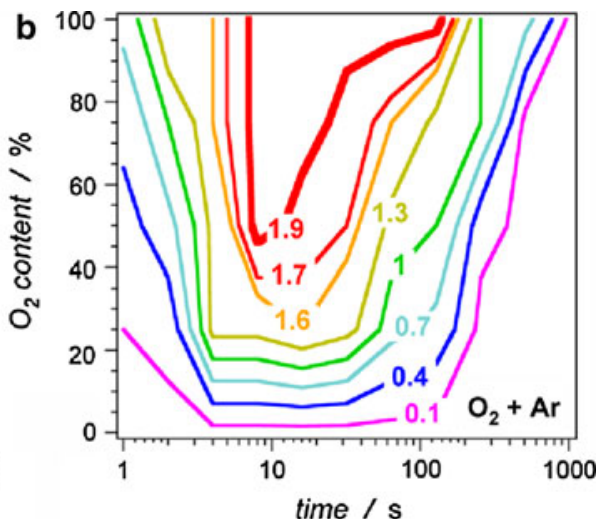

pressure $=0.35$ mbar; $R F$ power $=50 \mathrm{~W}$; and $R F=13.56 \mathrm{MHz}$ ). The contours corresponding to $\mathrm{QA}=1.7$ and 1.9 surround regions of conditions that can prove feasible for fabrication procedures

though we tend to eliminate the short-time treatment uncertainties by conducting blank runs and presetting $C_{\mathrm{Sh}}$ and $C_{\mathrm{Sr}}$ close to their optimal values, potential operator or instrument-setup errors make it unfeasibly for recommendation treatment times shorter than $10 \mathrm{~s}$ for these types of experiments.

A principal disadvantage of setting guidelines for using air as an oxidant is the seasonal and geographic inconsistency of the atmospheric content (such as humidity). Examination of different oxidizing gas mixtures indicated that plasma of pure oxygen provided the most acceptable set of conditions for achieving QA > 1.9 (Fig. 7). At $50 \mathrm{~W}$ RF power, treatment with oxygen plasma allows for time window between about 10 and $100 \mathrm{~s}$ (Table 1), which is wider than the treatment with air plasma under the same settings (Figs. 4b middle curve vs. Fig. 6 top curve). An increase in the RF power to $100 \mathrm{~W}$ or a decrease to $10 \mathrm{~W}$, however, compromises the quality of PDMS treatment with oxygen plasma (Millare et al. 2008). Treatments with air plasma, on the other hand, appeared to be less susceptible to such power fluctuations (Fig. 4b, top and bottom curves).

\section{Conclusions}

A semi-quantitative ternary analysis allowed us to survey a series of plasma-treatment conditions that yield permanent adhesion between PDMS and glass substrates. Oxygen plasma, generated at megahertz frequency (i.e., RF $=$ $13.56 \mathrm{MHz}, 50 \mathrm{~W}$ ), provided the preferred controllable means for oxidative treatment of PDMS surfaces in order to reliably obtain permanent adhesion with quantitative yields. We believe that our finding for PDMS-glass adhesion can be readily expanded to optimizing the procedure for adhering PDMS to other oxide substrates. 


\section{Experimental}

\subsection{Materials}

Precleaned microscope glass slides (Corning, $75 \mathrm{~mm} \times$ $50 \mathrm{~mm}$ ) were purchased from Fisher Scientific and cut into $10 \mathrm{~mm} \times 10 \mathrm{~mm}$ pieces. For the plasma, compressed gasses, oxygen (>99.5\%), nitrogen (>99.97\%), argon $(99.995 \%)$ were used.

Prepolymer of PDMS (Sylgard 184 silicone elastomer base kit) was purchased from Dow Corning Corporation. The PDMS prepolymer was mixed with the curing agent, degassed, poured into the polystyrene Petri dish (used for molds), and allowed to cure at $50^{\circ} \mathrm{C}$ for about $60 \mathrm{~min}$. The $3-\mathrm{mm}$ thick slabs were cut into rectangular pieces with areas about $1-2 \mathrm{~cm}^{2}$ (Millare et al. 2008).

\subsection{Plasma generation}

The plasma treatments were conducted using a capacitively coupled discharge systems (FEMTO, Diener Electronics) with 21 gas chambers equipped with a stainless-steal shelve. One of the systems was equipped with a $40-\mathrm{kHz} \mathrm{RF}$ generator (0-100 W variable power) and the other was equipped with a $13.56-\mathrm{MHz}$ RF generator (0-100 W variable power). The fully automated (with computer control) 13.56-MHz system had two gas inlets equipped with massflow controllers for regulating the composition of the binary gas mixtures.

For each set of gas mixtures, the plasma was ignited without samples in the chambers, and the shunt and series capacitors were tuned to manually and automatically for maximizing the transfer of the power to the ionized gas. The maximizing of the transfer of RF power was monitored via the computer control and/or via the intensity of the color of the plasma (Fig. 1). Thus, the obtained values for the shunt and series capacitances were preset for the sample-treatment procedures, so that the maximum power transfer is achieved immediately after the ignition of the plasma (with minimal or no capacitance shimming). Such presetting of the impedance-matching modules of the plasma systems is particularly important for the reproducibility of the short-duration treatments (i.e., for treatments shorter than about $5 \mathrm{~s}$ ).

\subsection{Plasma treatment}

The cut PDMS slabs and glass slides were placed into the plasma chamber and flushed with selected gases using conditions described previously (Millare et al. 2008). By controlling the flow rates from the two inlets via the mass flow controller, binary mixtures of oxygen-nitrogen and of oxygen-argon with various compositions were produced.
The gas pressure, in the sealed gas-flushed chamber, was brought down to 0.35 mbar and kept for about a minute allowing it to stabilize, at which point the RF generator was activated.

Post-plasma treatment, the samples were flushed with the selected gas, immediately placed on top of glass slides and cured under vacuum (340 mbar) using conditions previously established (Millare et al. 2008). The plasmatreated PDMS and glass surfaces were brought into contact within 10 - $30 \mathrm{~s}$ after the treatment. We observed that leaving the plasma treated substrates in air prior to bonding them for times as short as $5 \mathrm{~min}$, resulted in decreased quality of adhesion. This observation was consistent with the expected rearrangement of the oxidized polymer chains at the PDMS surface (Morra et al. 1990; Murakami et al. 1998).

\subsection{Examination of QA semi-quantitatively}

The QA of PDMS slabs adhered to glass slides was analyzed by applying relatively small lifting force, i.e., $1 \mathrm{~N}$ or less, at the edges (i.e., peeling forces). For each QA, the extent of bonded areas (i.e., permanent vs. reversible) were evaluated using previously established methods (Millare et al. 2008).

The data analysis was conducted using IgorPro v. 6 installed on MacOS and Windows workstations (Thomas et al. 2010c; Jones and Vullev 2002; Vullev and Jones 2002; Wan et al. 2008; Bao et al. 2009; Jones et al. 2007).

\subsection{Examination of QA via TSMs}

PDMS-glass adhesion stress-displacement experiments were conducted using a 100Q500 Universal Testing System (TestResources). The tested samples were prepared by sandwiching PDMS between two glass slides which were glued to wood blocks: i.e., wood/glass/PDMS/glass/wood junctions (Fig. 2a), where one side of the PDMS is always adhered to glass with $\mathrm{QA}=2$ and the other side being the examined QA. The stress-displacement studies yielded similar results as the previously reported observations for the different QA conditions (Millare et al. 2008). Because the tensile moduli of glass $(>\sim 50 \mathrm{GPa})$ and wood $(\sim 10 \mathrm{GPa})$ are considerably larger than the modulus of PDMS, the measured tensile strain (prior to the junction failure) was ascribed to deformation of PDMS. Therefore, the engineering stress, $\sigma$, was calculated from the applied tensile force and the areas of PDMS-glass contacts (prior to the measurements); and the engineering strain, $\varepsilon$, was estimated from the measured tensile displacement and the initial thickness of the PDMS slab. From TSM curves, prior to the failure of the junctions with QA $=2$ at both PDMS- 
glass interfaces, we estimated tensile moduli for PDMS of about $10 \mathrm{MPa}$.

Acknowledgments This research was supported by the National Science Foundation (CBET 0935995 and DBI 0731660) and by the U.C. Regents Faculty Development Award.

Open Access This article is distributed under the terms of the Creative Commons Attribution Noncommercial License which permits any noncommercial use, distribution, and reproduction in any medium, provided the original author(s) and source are credited.

\section{References}

Anderson JR, Chiu DT, Jackman RJ, Cherniavskaya O, McDonald JC, Wu H, Whitesides SH, Whitesides GM (2000) Fabrication of topologically complex three-dimensional microfluidic systems in PDMS by rapid prototyping. Anal Chem 72(14):3158-3164

Bao D, Millare B, Xia W, Steyer BG, Gerasimenko AA, Ferreira A, Contreras A, Vullev VI (2009) Electrochemical oxidation of ferrocene: a strong dependence on the concentration of the supporting electrolyte for nonpolar solvents. J Phys Chem A 113(7):1259-1267

Bar G, Delineau L, Hafele A, Whangbo MH (2001) Investigation of the stiffness change in, the indentation force and the hydrophobic recovery of plasma-oxidized polydimethylsiloxane surfaces by tapping mode atomic force microscopy. Polymer 42(8): 3627-3632

Belanger M-C, Marois Y (2001) Hemocompatibility, biocompatibility, inflammatory and in vivo studies of primary reference materials low-density polyethylene and polydimethylsiloxane: a review. J Biomed Mater Res 58(5):467-477

Bhattacharya S, Datta A, Berg JM, Gangopadhyay S (2005) Studies on surface wettability of poly(dimethyl) siloxane (PDMS) and glass under oxygen-plasma treatment and correlation with bond strength. J Microelectromech Syst 14(3):590-597

Chaudhury MK, Whitesides GM (1992) Correlation between surface free energy and surface constitution. Science 255(5049): $1230-1232$

Chueh B-H, Huh D, Kyrtsos CR, Houssin T, Futai N, Takayama S (2007) Leakage-free bonding of porous membranes into layered microfluidic array systems. Anal Chem 79(9):3504-3508

Cong H, Pan T (2008) Photopatternable conductive PDMS materials for microfabrication. Adv Funct Mater 18(13):1912-1921

Dittrich PS, Manz A (2006) Lab-on-a-chip: microfluidics in drug discovery. Nat Rev Drug Discov 5(3):210-218

Eddington DT, Puccinelli JP, Beebe DJ (2006) Thermal aging and reduced hydrophobic recovery of polydimethylsiloxane. Sens Actuators B 114(1):170-172

Gates BD (2005) Nanofabrication with molds and stamps. Mater Today 8(2):44-49

Ginn BT, Steinbock O (2003) Polymer surface modification using microwave-oven-generated plasma. Langmuir 19(19):81178118

Gong X, Wen W (2009) Polydimethylsiloxane-based conducting composites and their applications in microfluidic chip fabrication. Biomicrofluidics 3(1):012007

Gou H-L, Xu J-J, Xia X-H, Chen H-Y (2010) Air plasma assisting microcontact deprinting and printing for gold thin film and PDMS patterns. ACS Appl Mater Interfaces 2(5):1324-1330

Haubert K, Drier T, Beebe D (2006) PDMS bonding by means of a portable, low-cost corona system. Lab Chip 6(12):1548-1549
Hillborg H, Ankner JF, Gedde UW, Smith GD, Yasuda HK, Wikstrom K (2000) Crosslinked polydimethylsiloxane exposed to oxygen plasma studied by neutron reflectometry and other surface specific techniques. Polymer 41(18):6851-6863

Hong C, Bao D, Thomas MS, Clift JM, Vullev VI (2008) Print-andpeel fabrication of microelectrodes. Langmuir 24(16):8439-8442

Hopwood J, Mantei TD (2003) Application-driven development of plasma source technology. J Vac Sci Technol A 21(5S):S139S144

Hui AYN, Wang G, Lin B, Chan W-T (2005) Microwave plasma treatment of polymer surface for irreversible sealing of microfluidic devices. Lab Chip 5(10):1173-1177

Jacob W (1998) Surface reactions during growth and erosion of hydrocarbon films. Thin Solid Films 326(1-2):1-42

Jones G II, Vullev VI (2002) Medium effects on the stability of terbium(III) complexes with pyridine-2,6-dicarboxylate. J Phys Chem A 106(35):8213-8222

Jones G II, Yan D, Hu J, Wan J, Xia B, Vullev VI (2007) Photoinduced electron transfer in arylacridinium conjugates in a solid glass matrix. J Phys Chem B 111(24):6921-6929

Kawamura E, Vahedi V, Lieberman MA, Birdsall CK (1999) Ion energy distributions in RF sheaths; review, analysis and simulation. Plasma Sources Sci Technol 8(3):R45-R64

Lee JN, Park C, Whitesides GM (2003) Solvent compatibility of poly(dimethylsiloxane)-based microfluidic devices. Anal Chem 75(23):6544-6554

Li N, Schwartz M, Ionescu-Zanetti C (2009) PDMS compound adsorption in context. J Biomol Screen 14(2):194-202

McDonald JC, Whitesides GM (2002) Poly(dimethylsiloxane) as a material for fabricating microfluidic devices. Acc Chem Res 35(7):491-499

Mehta G, Lee J, Cha W, Tung Y-C, Linderman JJ, Takayama S (2009) Hard top soft bottom microfluidic devices for cell culture and chemical analysis. Anal Chem 81(10):3714-3722

Millare B, Thomas M, Ferreira A, Xu H, Holesinger M, Vullev VI (2008) Dependence of the quality of adhesion between polydimethyl siloxane and glass surfaces on the conditions of treatment with oxygen plasma. Langmuir 24(22):1321813224

Morra M, Occhiello E, Marola R, Garbassi F, Humphrey P, Johnson D (1990) On the aging of oxygen plasma-treated polydimethylsiloxane surfaces. J Colloid Interface Sci 137(1):11-24

Murakami T, Kuroda S-i, Osawa Z (1998) Dynamics of polymeric solid surfaces treated with oxygen plasma: effect of aging media after plasma treatment. J Colloid Interface Sci 202(1):37-44

Salem MM, Loiseau JF, Held B (1998) Impedance matching for optimization of power transfer in a capacitively excited radiofrequency plasma reactor. Eur Phys J Appl Phys 3(1):91-95

Shin YS, Cho K, Lim SH, Chung S, Park S-J, Chung C, Han D-C, Chang JK (2003) PDMS-based micro pcr chip with parylene coating. J Micromech Microeng 13(5):768-774

Singh KP, Roy S (2007) Impedance matching for an asymmetric dielectric barrier discharge plasma actuator. Appl Phys Lett 91(8):081504/081501-081504/081503

Thomas MS, Clift JM, Millare B, Vullev VI (2010a) Print-and-peel fabricated passive micromixers. Langmuir 26(4):2951-2957

Thomas MS, Millare B, Clift JM, Bao D, Hong C, Vullev VI (2010b) Print-and-peel fabrication for microfluidics: what's in it for biomedical applications? Ann Biomed Eng 38(1):21-32

Thomas MS, Nunez V, Upadhyayula S, Zielins ER, Bao D, Vasquez JM, Bahmani B, Vullev VI (2010c) Kinetics of bacterial fluorescence staining with $3,3^{\prime}$-diethylthiacyanine. Langmuir 26(12):9756-9765

Vullev VI, Jones G (2002) Photoinduced electron transfer in alkanoylpyrene aggregates in conjugated polypeptides. Tetrahedron Lett 43(47):8611-8615 
Vullev VI, Wan J, Heinrich V, Landsman P, Bower PE, Xia B, Millare B, Jones G II (2006) Nonlithographic fabrication of microfluidic devices. J Am Chem Soc 128(50):16062-16072

Wan J, Ferreira A, Xia W, Chow CH, Takechi K, Kamat PV, Jones G, Vullev VI (2008) Solvent dependence of the charge-transfer properties of a quaterthiophene-anthraquinone dyad. J Photochem Photobiol A 197(2-3):364-374

Whitesides GM (2006) The origins and the future of microfluidics. Nature 442(7101):368-373
Zhou J, Ellis AV, Voelcker NH (2010) Recent developments in PDMS surface modification for microfluidic devices. Electrophoresis 31(1):2-16

Zhuang X, Nikoozadeh A, Beasley MA, Yaralioglu GG, Khuri-Yakub BT, Pruitt BL (2007) Biocompatible coatings for CMUTs in a harsh, aqueous environment. J Micromech Microeng 17(5): 994-1001 